\title{
miR-16-1-3p targets TWIST1 to inhibit cell proliferation and invasion in NSCLC
}

\author{
Feng $\mathrm{QQ}^{1 *}$, Dong $\mathrm{ZQ}^{1 *}$, Zhou $\mathrm{Y}^{1}$, Zhang $\mathrm{H}^{1}$, Long $\mathrm{C}^{2}$ \\ Department of Respiratory Medicine, The Second Clinical Medical College, Yangtze University, \\ Jingzhou, Hubei, China. conglong523@sina.com
}

\begin{abstract}
In our study, the impact of miR-16-1-3p on cell proliferation and invasion in NSCLC was explored. miR-16-1$3 p$ mimics were transfected to A549 cells for miR-16-1-3p overexpression. qRT- PCR and Western blot were applied to explore the relative expression of mRNA and protein in A549 cells. Furthermore, the cell proliferation capability was determined by MTT assay. Additionally, cell migration and invasion were measured using a scratch assay and transwell assay, respectively. Moreover, TargetScan and luciferase reporter assay was utilized to investigate the target of miR-16-1-3p. The results indicated that miR-16-1-3p was downregulated in NSCLC cells and upregulation of miR-16-1-3p was able to inhibit the expression of TWIST1. In addition, the reduced cell proliferation, inhibited cell migration and invasion were observed in miR-16-1-3p mimic group compared to the negative control group. The luciferase reporter gene showed that TWIST1 was a target of miR-16-1-3p. Therefore, the present study demonstrated that miR-16-1-3p may suppress A549 cell proliferation, migration and invasion by targeting TWIST1. Thus, miR-16-1-3p might play important roles in NSCLC development, which provides a novel aspect for NSCLC investigation (Fig. 6, Ref. 26). Text in PDF www.elis.sk.

KEY WORDS: miR-16-1-3p, TWIST1, NSCLC, proliferation, invasion, target.
\end{abstract}

\section{Introduction}

Lung cancer is the leading cause of cancer death worldwide. Moreover, the overall 5-year survival rate of lung cancer was about $5 \%$ due to a late diagnosis. One third of the patients is diagnosed with stage III disease $(1,2)$. It is reported that approximately $80 \%$ of lung cancer refers to non-small cell lung cancer (NSCLC), which includes adenocarcinoma (AC), squamous cell carcinoma (SQCC) and large cell carcinoma (LCLC) (3). The development of NSCLC involves a series of genetic and epigenetic alterations, which ultimately becomes the invasive cancer via clonal expansion. In addition, the process of invasion, migration and resistance to cancer therapy is related to the accumulation of genetic and epigenetic alterations (4). Generally, the treatment of NSCLC refers to surgery, chemotherapy, radiation therapy or a combined modality approach (5). Although the molecularly targeted therapies advent has significantly improved the outcomes for patients, the prolonged disease control and increased overall 5-year survival rate is still poor (6).

\footnotetext{
${ }^{1}$ Department of Respiratory Medicine, The Second Clinical Medical College, Yangtze University, Jingzhou, Hubei, China, and ${ }^{2}$ Department of Clinical Laboratory, The First Affiliated Hospital of Yangtze University, Jingzhou, Hubei, China

Address for correspondence: C. Long, Dr, Department of Clinical Laboratory, The First Affiliated Hospital of Yangtze University, No. 8 Hangkong Road, Jingzhou, Hubei, 434100, China.

Acknowledgement: We thank Yangtze University and the First Affiliated Hospital of Yangtze University for equipment and tissues supporting.

*These authors contributed equally.
}

MicroRNAs play vital roles in mRNA silencing at the posttranslational level. MiRNAs are able to cleave the mRNA strand into pieces through RISC, shorten the poly(A) tails of mRNA to destabilize mRNA or interfere the process of translation from mRNA to protein $(7,8)$. miRNA can not only be used for early detection of lung cancer and screening, but also for the treatment of lung cancer. Due to the function of miRNA in metastasis prevention, radiation therapy and chemotherapy resistance reduction in lung cancer, miRNAs are regarded as novel promising players in lung cancer (9). The oncogenic miRNAs in lung cancers include miR-17 92 cluster, miR-21, miR-221/222 and so on. The tumor suppressive miRNAs in lung cancer are let-7, miR-34/449, miR-15/16, miR-200, miR-205 and miR-143/145 and so forth (7).

Twist-related protein 1(TWIST1), known as class A basic helix-loop-helix protein 38 (bHLHa38), is a basic helix-loop-helix transcription factor, which is essential for the mesoderm formation in drosophila and mouse. Furthermore, TWIST1 can promote epithelial-mesenchymal transition (ETM) process (10). Some studies reported that the DNA methylation of TWIST1 gene was associated with cancer cell malignant progression, invasion and metastasis, while the underlying mechanism is not fully understood. $(11,12)$. In gastric cancer and breast cancer, upregulation of TWIST1 promotes the cell invasion and metastasis $(10,13)$. However, the roles of TWIST1 in NSCLC have not been widely explored. Therefore, we hypothesized that some miRNAs may target TWIST1 in NSCLC. According to TargetScan and miRBase website, we selected miR-16-1-3p, which can target TWIST1 in gastric cancer as our target (13). 
As we all know, N-cadherin is upregulated in many cancers, which play key roles in tumor cell movement and ETM process (14). The overexpression of N-cadherin contributes to the metastasis and invasion of tumor cells (15). Additionally, $\alpha$-SMA and Fibronectin are also related to lung fibrosis and ETM process (16). Therefore, the expression of N-cadherin, $\alpha$-SMA and Fibronectin were measured in our study.

\section{Materials and methods}

Cancer tissues and paracarcinoma tissues of NSCLC

We collected 60 paraffin specimens (30 cancer tissues and 30 paracarcinoma tissues) of NSCLC from patients, who underwent surgery at the First Affiliated Hospital of Yangtze University from March, 2016 to May, 2017. Additionally, all specimens were stored in liquid nitrogen in EP tubes and all patients were given the informed consent. The present study was approved by the Ethics Committee of the First Affiliated Hospital of Yangtze University prior to the study.

\section{Cell lines}

A549 and H1650 (SL003 and CRL5883, ATCC) were regarded as the cancer cell lines and the BEAS-2B cell lines (CRL9609, ATCC) as the internal reference. The selected cells were maintained in RPMI 1640 medium supplemented with $10 \%$ fetal bovine serum (31800, Beijing solarbio science\&technology Co., Ltd., Beijing, China) and $200 \mu / \mathrm{ml}$ Gentamicin cultured in a humidified incubator at $37^{\circ} \mathrm{C}$ with $5 \% \mathrm{CO}_{2}$.

\section{Target prediction}

TargetScan (http://www.targetscan.org/vert_71/) and miRWalk ( http://zmf.umm.uni-heidelberg.de/apps/zmf/mirwalk/predictedmirnagene.html) were applied for target gene prediction

qRT-PCR for miR-16-1-3p expression in NSCLC tissues and cells

qRT-PCR was employed to study the expression levels of miR-16-1-3p in NSCLC tissues and cells. Firstly, QuickExtract ${ }^{\mathrm{TM}}$ RNA Extraction Kit (QER090150, Epicentre) was applied for RNA extraction from tissues and cell lines according to manufacturer's instructions. PrimeScriptTM RT-PCR kit (RR014A, TaKaRa) was used for RT-PCR reaction. The reaction conditions of RT-PCR were set by $96^{\circ} \mathrm{C}$ for $5 \mathrm{~min}, 95^{\circ} \mathrm{C}$ for $30 \mathrm{~s}, 60^{\circ} \mathrm{C}$ for $30 \mathrm{~s}$, and $76{ }^{\circ} \mathrm{C}$ for $1.5 \mathrm{~min}$ for 32 cycles, $4{ }^{\circ} \mathrm{C}$ for storage. Moreover, U6 was regarded as the internal reference. Additionally, MystiCq ${ }^{\circledR}$ microRNA qPCR Assay Primer hsa-miR-16-1-3p (MIRAP0030, Sigma-Aldrich) was used as the miR-16-1-3p primer. The $2^{-\Delta \Delta C q}$ method was used to analyze the mRNA expression (17).

\section{Cell transfection}

In order to explore the function of miR-16-1-3p in NSCLC cells, A549 cell line was selected as our experimental cells. A549 cells were placed in the 24-well plates and incubated in RPMI 1640 medium at $37{ }^{\circ} \mathrm{C}$ with $5 \% \mathrm{CO}_{2}$. miR-16-1-3p mimic plasmids (500 ng) and mimic control plasmids (500 ng) were transfected to A549 cells using $2.5 \mu 1$ Lipofectamine 2000 (Invitrogen). Fur- thermore, miR-16-1-3p mimic plasmids and mimic control plasmids were purchased from CYAGEN Biotechnology, Co. Ltd., Suzhou, China. After $6 \mathrm{~h}$ incubation, the medium was replaced by $10 \%$ bovine serum fresh culture.

\section{Western Blot}

qRT-PCR and western blot was performed to study the expression levels of TWIST1, N-cadherin, alpha-SMA and Fibronectin profile in transfected A549 cells. For relative expression of mRNA, RNeasy Mini Kit (74104, Qiagen, German) was utilized to isolate RNA according to manufacturer's protocol. Then, PrimeScriptTM RT-PCR kit (RR014A, TaKaRa) was used for RT-PCR reaction.

For western blot, transfected A549 cells were lysed by RIPA (P0013B, Beyotime Biotechnology Co. Ltd., Nanjing, China) and then centrifuged at $10,000 \mathrm{rpm}$ for $15 \mathrm{~min}$ at $4{ }^{\circ} \mathrm{C}$. GAPDH was regarded as the internal control. Anti-TWIST1 (2F8E7, 1:500), -N-cadherin (33-3390, 1:500), -alpha-SMA (1A4, 1:100), -Fibronectin (FBN, 1:500), -GAPDH (GA1R, 1:500) and the secondary antibodies $(35518,1: 5000)$ were purchased from Thermo Fisher Scientific. Furthermore, ECL Western blotting kit (K820500, Biovision Inc., USA) was adopted for membrane stain. Gray value was obtained from ImageJ image analysis software. This experiment was performed in triplicate. The antibodies incubation was based on Wang Tao's research (13).

\section{MTT assay}

After transfection, the A549 cells were placed into 24-well plates and the MTT stain was performed at $24 \mathrm{~h}, 48 \mathrm{~h}$ and $72 \mathrm{~h}$ using MTT Cell Viability Assay Kit (KA1606, Abnova Corporation). The experiments were performed according to Lei Li's research (18).

\section{Scratch assay}

Scratch assay was employed to study the migration of A549 cells after transfection. A549 cells $\left(5 \times 10^{5}\right)$ were placed into $24-$ well plates and cultured for $24 \mathrm{~h}$ to obtain a monolayer. Then, the scratch was made across the center of the well using the tip remaining perpendicular to the bottom of the well. After that, the cells were incubated with RPMI 1640 medium at $37{ }^{\circ} \mathrm{C}$ with $5 \%$ $\mathrm{CO}_{2}$ for $24 \mathrm{~h}$. Images of the migrated cells were captured at $24 \mathrm{~h}$ using ImageJ.

\section{Transwell assay}

Transwell assay was performed to identify the invasion ability of A549 cells after transfection. Transwell culture inserts (8-mm pore size; Falcon; BD Biosciences) were placed into the wells of 24-well culture plates with separate upper and lower chambers. The main procedures were based on the research of Lei Li (18).

\section{Luciferase reporter assay}

The target verification was performed by luciferase reporter assay. The constructed TWIST1 3'-UTR wild and TWIST1 3'-UTR mutant plasmids were purchased from Vigene Bioscience Co. Ltd. Subsequently, the luciferase activity was measured by luciferase assay kit (GM-040502A, Qcbio Science \& Technologies Co. Ltd., China.) and fluorescence microscopy (13). 

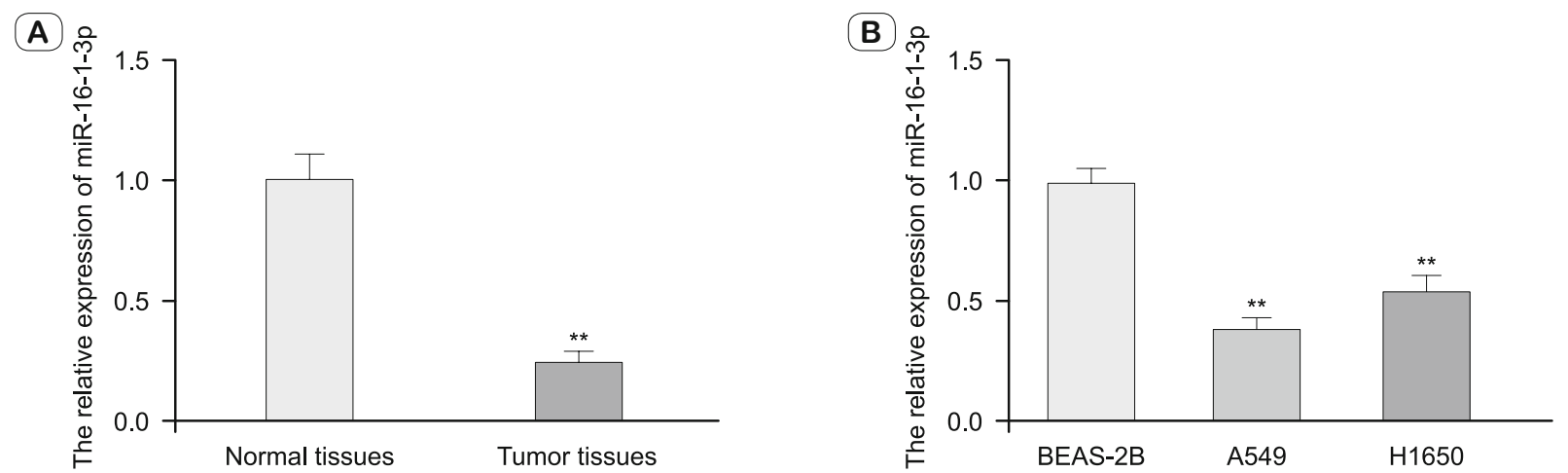

Fig. 1. The relative expression of miR-16-1-3p in NSCLC tissues and cells was measured by qRT-PCR. Moreover, a decreased expression of miR-16-1-3p was observed in NSCLC cancer tissues, A549 and H1650 cell lines compared to normal tissues and BEAS-2B cell lines respectively. A) The relative expression of miR-16-1-3p in tumor tissues and normal tissues. B) The relative expression of miR-16-1-3p in A549, H1650 and BEAS-2B cell lines. ${ }^{* *} \mathrm{p}<0.01$.

\section{Statistical method}

All quantitative data were presented as the mean \pm standard deviation (SD). Furthermore, the expression abundance of miRNA and mRNA was identified by $2^{-\triangle \Delta C q}$ method. Statistical analysis was performed using GraphPad Prism version 5.01 (GraphPad Software, Inc., La Jolla, CA, USA) via Student t test. For all comparisons, $\mathrm{p}<0.05$ was considered to indicate significant differences.

\section{Results}

miR-16-1-3p expressions in NSCLC cancer and normal tissues and cells

The relative expression of miR-16-1-3p in NSCLC tissues and cells (A549, H1650 and BEAS-2B) was determined by qRTPCR. The results implied that miR-16-1-3p was downregulated in NSCLC tumor tissues compared to paracarcinoma tissues, $p$ $<0.01$ (Fig. 1A). Furthermore, the expression of miR-16-1-3p was significantly decreased in A549 and $\mathrm{H} 1650$ cells than that of BEAS-2B cell, $p<0.01$ (Fig. 1B). The results of of miR-16-1-3p expression in cancer tissues and cell lines were consistent.

\section{Expression of miR-16-1-3p in transfected cells}

To further investigate the function of miR-16-1-3p in NSCLC, A549 cell line was selected for the following experiments. A549 cells were transfected with miR-16-1-3p mimic plasmids and mimic control plasmids. As shown in Figure 2, the expression of miR-16-1-3p significantly increased in miR-16-1-3p mimic group versus negative control group, $\mathrm{p}<0.01$. Therefore, the transfection was successful.

m RNA and protein expression of TWIST1, N-cadherin, alphaSMA, Fibronectin

After transfection, A549 cells were cultured for $72 \mathrm{~h}$ at 37 ${ }^{\circ} \mathrm{C}$ with $5 \% \mathrm{CO}_{2}$. qRT-PCR and Western blot was applied for relative expression of mRNAs and proteins. In Figure 3A, the results indicated that the expression of mRNA of protein TWIST1,

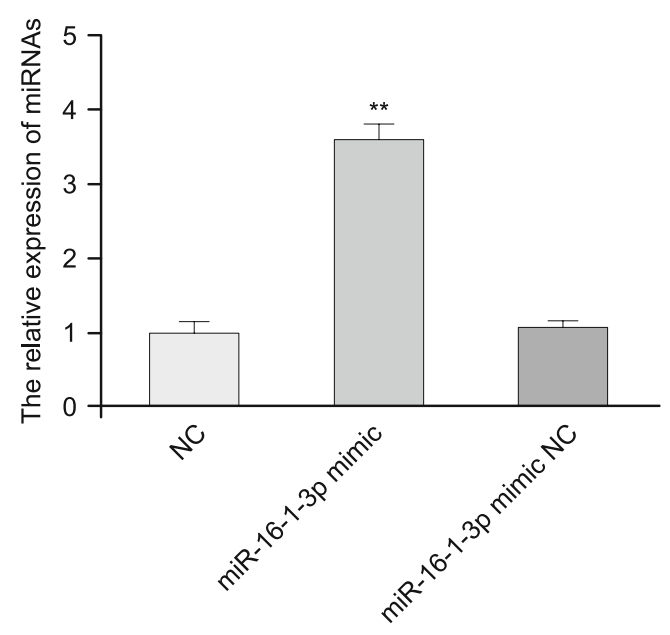

Fig. 2. miR-16-1-3p mimic and mimic control plasmids were transfected to $A 549$ cells and the expression of miR-16-1-3p was quantified by qRT-PCR. The relative expression of miR-16-1-3p was shown in Figure 2. NC - negative control; miR-16-1-3p mimic: cells were transfected with miR-16-1-3p mimic plasmids; miR-16-1-3p mimic NC - cells were transfected with mimicking negative control plasmids. ${ }^{* *} \mathbf{p}<0.01$.

N-cadherin, alpha-SMA and Fibronectin was dramatically downregulated in miR-16-1-3p mimic group compared to the negative control groups $(\mathrm{p}<0.01)$. Moreover, the expression of TWIST1, $\mathrm{N}$-cadherin, alpha-SMA and Fibronectin protein was suppressed in miR-16-1-3p mimic group compared to the negative control groups $(p<0.01)$ (Figs 3B,C). Therefore, miR-16-1-3p might inhibit the expression of TWIST1, N-cadherin, alpha-SMA and Fibronectin.

\section{MTT assay}

The A549 cell proliferation profile was measured by MTT stain after $24 \mathrm{~h}, 48 \mathrm{~h}$ and $72 \mathrm{~h}$ incubation. In addition, the OD value was identified by microplate reader under $490 \mathrm{~nm}$ wavelength. The increased cell proliferation was observed in miR-16-1-3p mimic groups versus negative control groups. Furthermore, the cell proliferation was significantly promoted the in mimic groups at $72 \mathrm{~h}$ incubation compared to the negative control groups $(\mathrm{p}<0.01)$ (Fig. 4). 

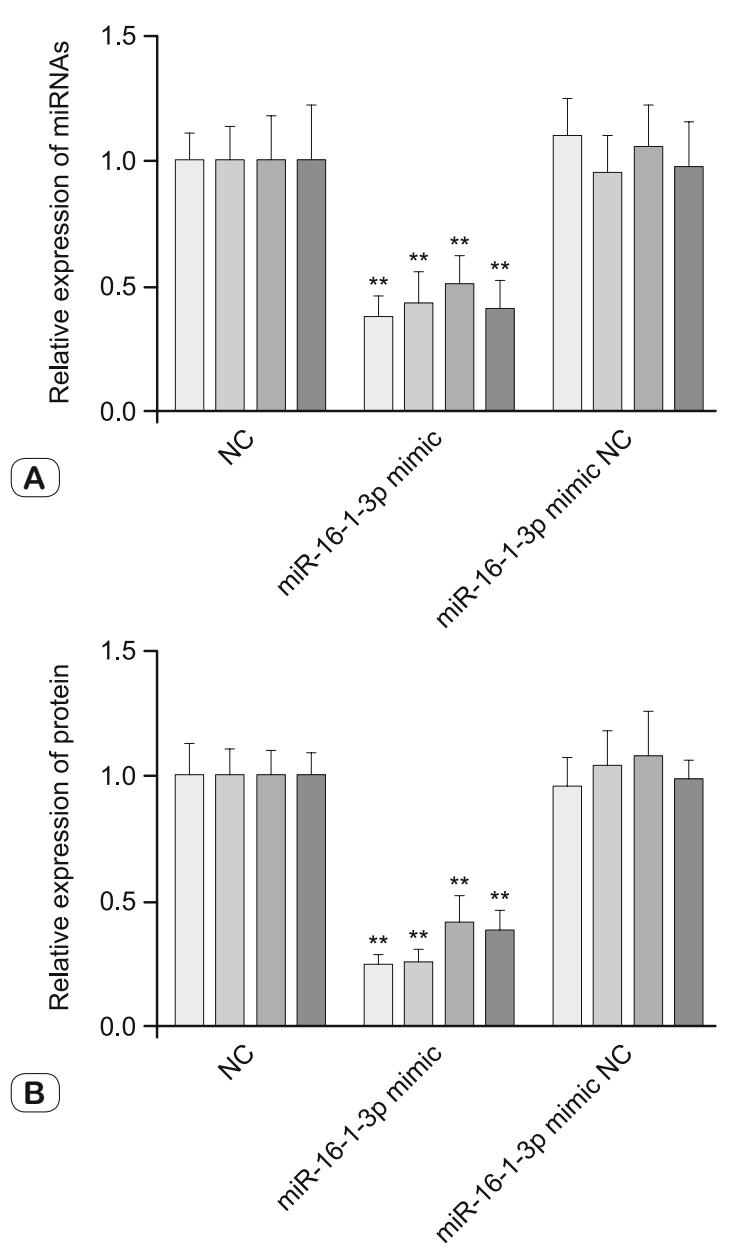

$\square$ Twist $1 \square$ N-cadherin $\square$ alpha-SMA $\square$ Fibronectin

(C)

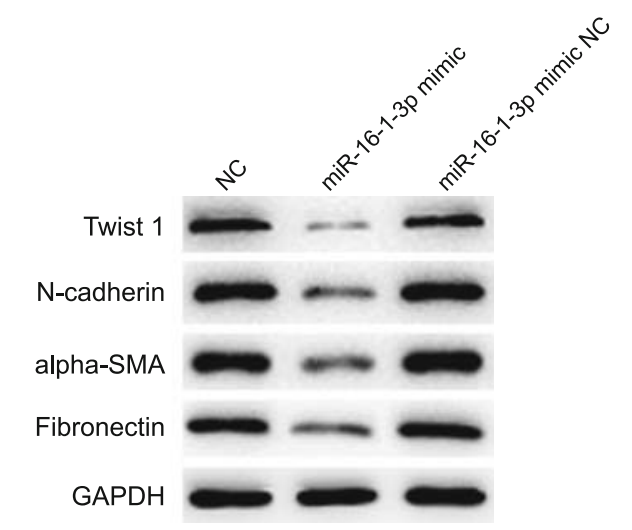

Fig. 3. 72 h incubation after transfection, cells were collected for mRNAs and protein expression via qRT-PCR and western blot. A) Relative expression of mRNAs of TWIST1, N-cadherin, alpha-SMA and Fibronectin. B) Relative expression of TWIST1, N-cadherin, alpha-SMA and Fibronectin. C) Expression of protein TWIST1, N-cadherin, alpha-SMA and Fibronectin determined by Western blot. NC - negative control; miR-16-1-3p mimic: cells were transfected with miR-16-1-3p mimic plasmids; miR-16-1-3p mimic NC - cells were transfected with mimic negative control plasmids. $* * \mathbf{p}<0.01$.

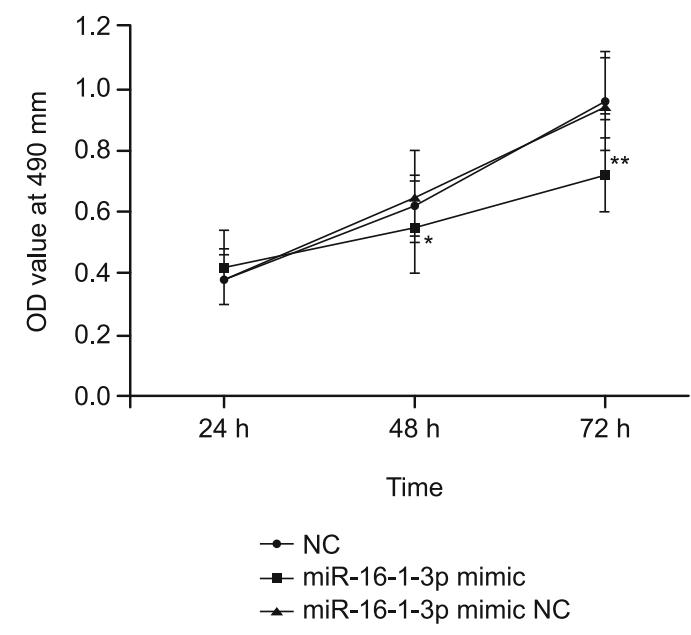

Fig. 4. A549 cells were transfected with miR-16-1-3p mimic and mimic control plasmids. Then, MTT assay was performed at time point $24 \mathrm{~h}$, $48 \mathrm{~h}$ and $72 \mathrm{~h}$. The cell viability was identified by OD value under 490 nm wavelength. The decreased OD490 value was observed in miR-161-3p mimic groups compared to mimic control groups. NC - negative control; miR-16-1-3p mimic: cells were transfected with miR-16-1-3p mimic plasmids; miR-16-1-3p mimic NC - cells were transfected with mimicking negative control plasmids. ${ }^{*} \mathbf{p}<0.05,{ }^{* *} \mathrm{p}<0.01$.

\section{Scratch assay and transwell assay}

Scratch assay and transwell assay were performed after transfection followed by $72 \mathrm{~h}$ incubation. As shown in Figure 5A, the results demonstrated that the migration ability of A549 cells was suppressed in miR-16-1-3p groups compared to the negative control groups due to the wider scratch in the mimic groups. Additionally, the decreased cell invasion was observed in miR16-1-3p mimic groups compared to negative control groups $(\mathrm{p}<$ 0.01 ) (Figs 5B,C). Hence, the inhibited cell migration and invasion capability of A549 cells might be caused by the overexpression of miR-16-1-3p.

Target gene prediction and luciferase reporter assay

Target gene of miR-16-1-3p, TWIST1, was identified by TargetScan as shown in Figure 6A. miR-16-1-3p mimic plasmids and mimic control plasmids were co-transfected with the TWIST1 3'URT-WT and 3'UTR-MUT plasmids. After $72 \mathrm{~h}$ incubation, the luciferase activity was measured by fluorescence microscopy. As shown in Figure 6B, a decreased luciferase activity was observed in miR-16-1-3p with TWIST1 3'-UTR-WT groups compared to the negative control groups $(\mathrm{p}<0.05)$. Therefore, TWIST1 was a target gene of miR-16-1-3p.

\section{Discussion}

In humans, TWIST1 malfunction was involved in SaethreChotzen syndrome. Later, the essential roles of TWIST1 in tumor initiation, development, invasion and metastasis along with the chemo resistance was identified in a variety of carcinomas (19). TWIST1, a basic helix-loop-helix (bHLH) transcription factor, 

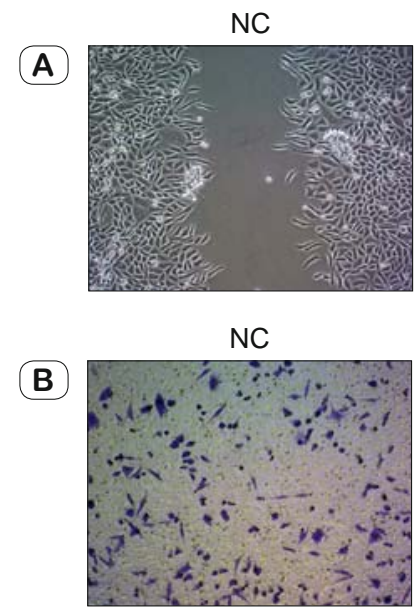

miR-16-1-3p mimic

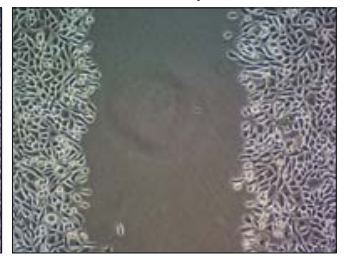

miR-16-1-3p mimic

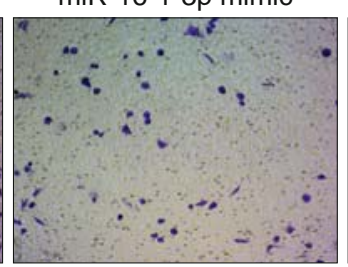

miR-16-1-3p mimic NC

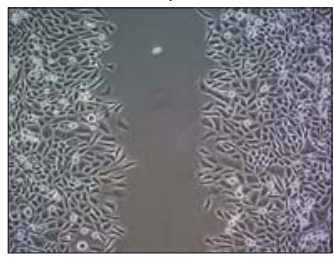

miR-16-1-3p mimic NC

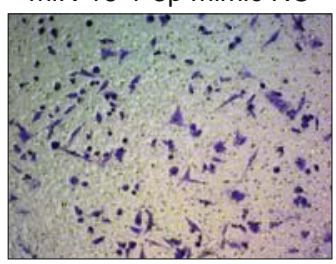

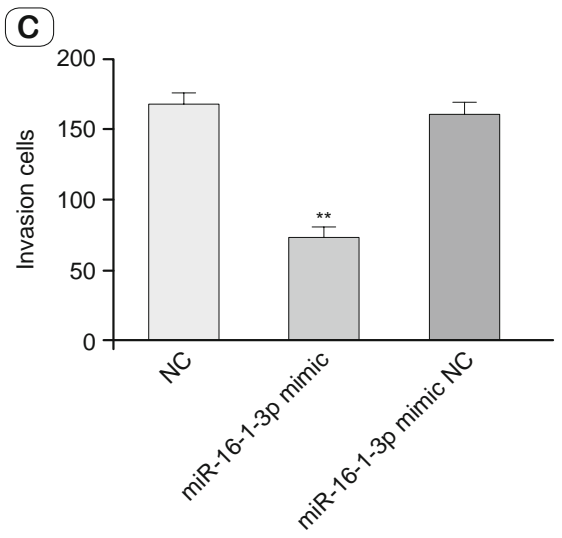

Fig. 5. $72 \mathrm{~h}$ incubation after transfection, A549 cells were collected for scratch and transwell assay. A) Results of scratch assay in NC, miR-16-13p mimic and mimic control groups; B) Results of transwell assay in NC, miR-16-1-3p mimic and mimic control groups; C) Histogram of cells invasion in NC, miR-16-1-3p mimic and miR-16-1-3p mimic control groups. NC - negative control; miR-16-1-3p mimic: cells were transfected with miR-16-1-3p mimic plasmids; miR-16-1-3p mimic NC - cells were transfected with mimicking negative control plasmids. $* *$ p $<0.01$.

miR-16-1-3p 3' AGUCGUCGUGUCAAUUAUGACC

TWIST1 $33^{\prime}$ ACCUUUAGAAAUAAAAAAUACUGG

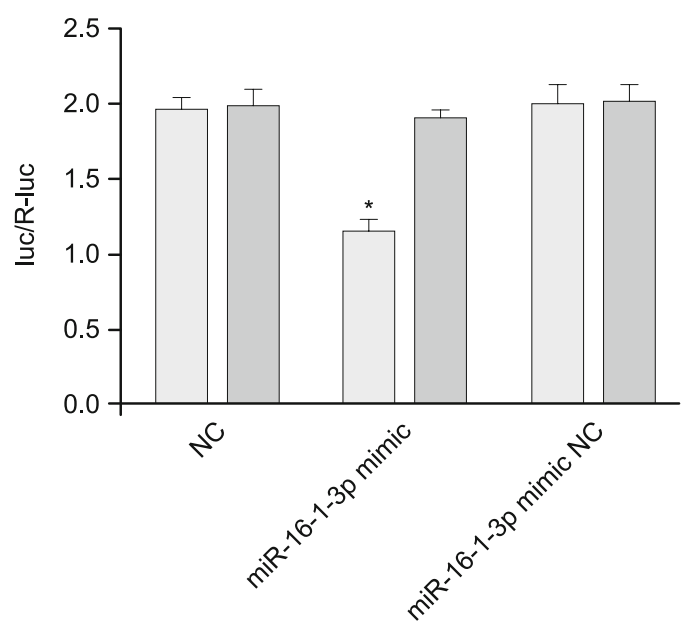

$\square$ 3' UTR-WT

$\square$ 3' UTR-MUT

Fig. 6. TargetScan and miRWalk were utilized for a target gene prediction. $72 \mathrm{~h}$ after transfection incubation, a luciferase reporter system for targeting verification. A) Sequence of miR-161-3p and TWIST1. B) Luciferase activity in NC, miR-16-1-3p mimic and mimic control groups. NC - negative control; miR-16-1-3p mimic: cells were transfected with miR-16-1-3p mimic plasmids; miR-16-1-3p mimic NC - cells were transfected with mimicking negative control plasmids. ${ }^{*} p<0.05$.

plays a significant role in promoting cancer cell metastasis and in the development of epithelial-mesenchymal transition (ETM) (10). Moreover, TWIST1 has four domains referring to a TWIST1 domain, a glycine rich region, bHLH domain and the Twist box (or WR domain) (20). The study showed that TWIST1 is involved in numerous signaling pathways concerning FGF and sonic hedgehog (SHH) pathways (20). Additionally, the results showed that the conserved Twist box domain is necessary in the context of cancer phenotypes (21). From the study of Lei Li, the expression of TWIST1 was upregulated and a knockdown of TWIST1 is able to inhibit the migration and invasion of NSCLC cell, which is consistent with our study (18). Additionally, the prognosis and chemo resistance of lung cancer was associated with the expression of TWIST1 in lung cancer (22). Therefore, in the future, the impact of miR-16-1-3p on TWIST1 regarding the prognosis and chemo resistance in NSCLC could be explored. Also, the study revealed that the expression of $\mathrm{N}$-cadherin was suppressed due to the overexpression of miR-32, which is in line with our present study (18). Whereas, the expression of vimentin and MMP-2 and MMP-9 were not explored, which should be further investigated.

A number of studies have found that abnormal expression of miRNAs was directly involved in the regulation of NSCLC development. For example, miR-378 played a key role in NSCLC cell growth, vascularization and metastasis in the study by Klaudia Skrzypek et al (23). Moreover, through next-generation deep sequencing, the microRNA transcriptome in lung cancer were characterized. The results showed that miR-944 might have the oncogenic function in the pathogenesis of NSCLC (24). Furthermore, five miRNAs (miR-20a, miR-223, miR-21, miR-221 and miR-145) may be novel biomarkers for screening early-stage NSCLC studied by Qing Geng et al (25). As we all know, miR-16, miR-15, miR-195 and miR-457 are related to microRNA precursor sequences from the miR-15 gene family (26). Therefore, the further study could target miR-15, miR-195 and miR-475 involving the correlation between these miRNAs and TWIST1.

In conclusion, the results implied that miR-16-1-3p was downregulated and TWIST1 was upregulated in NSCLC. Moreover, decreased TWIST1 expression was observed due to an overexpression of miR-16-1-3p. Also, the luciferase reporter assay identified that TWIST1 was the direct target of miR-16-1-3p. Furthermore, 
the inhibited cell proliferation and invasion showed that miR16-1-3p was able to inhibit NSCLC development by targeting TWIST1. These findings may provide a novel target for NSCLC development exploration.

\section{References}

1. Dhanasekaran SM, Balbin OA, Chen G et al. Transcriptome MetaAnalysis of Lung Cancer Reveals Recurrent Aberrations in NRG1 and Hippo Pathway Genes. Nature communications 2014; 5: 5893.

2. Askoxylakis V, Tanner J, Kappes $\mathbf{J}$ et al. Trimodal therapy for stage III-N2 non-small-cell lung carcinoma: a single center retrospective analysis. BMC Cancer 2014; 14: 572.

3. Shtivelman E, Hensing T, Simon GR et al. Molecular pathways and therapeutic targets in lung cancer. Oncotarget 2014; 5 (6): 1392-1433.

4. Lemjabbar-Alaoui H, Hassan O, Yang Y-W, Buchanan P. Lung cancer: biology and treatment options. Biochimica Bioph Acta 2015; 1856 (2): $189-210$

5. Farhat FS, Houhou W. Targeted therapies in non-small cell lung carcinoma: what have we achieved so far? Ther Adv Med Oncol 2013; 5 (4): 249-270.

6. Anagnostou VK, Brahmer JR. Cancer Immunotherapy: A Future Paradigm Shift in the Treatment of Non-Small Cell Lung Cancer. Clin Cancer Res 2015; 21 (5): 976-984.

7. Qi J, Mu D. MicroRNAs and lung cancers: from pathogenesis to clinical implications. Front Med 2012; 6 (2): 134-155.

8. Bartel DP. MicroRNA Target Recognition and Regulatory Functions. Cell 2009; 23; 136 (2): 215-233.

9. Tufman A, Tian F, Huber RM. Can MicroRNAs Improve the Management of Lung Cancer Patients? A Clinician's Perspective. Theranostics 2013; 3 (12): 953-963.

10. Xu Y, Qin L, Sun T et al. TWIST1 Promotes Breast Cancer Invasion and Metastasis by Silencing Foxal Expression. Oncogene 2017; 36 (8): 1157-1166.

11. Tan J, Tedrow JR, Nouraie M et al. Loss of TWIST1 in the Mesenchymal Compartment Promotes Increased Fibrosis in Experimental Lung Injury By Enhanced Expression of C-X-C Motif Ligand 12 (CXCL12). J Immunol (Baltimore), 1950; 198 (6): 2269-2285.

12. Sakamoto A, Akiyama Y, Shimada S, Zhu W-G, Yuasa Y, Tanaka S. DNA Methylation in the Exon 1 Region and Complex Regulation of TWIST1 Expression in Gastric Cancer Cells. Roh T-Y, ed. PLoS ONE 2015; 10 (12): e0145630.

13. Wang T, Hou J, Li Z et al. miR-15a-3p and miR-16-1-3p Negatively Regulate TWIST1 to Repress Gastric Cancer Cell Invasion and Metastasis. Internat J Biol Sci 2017; 13 (1): 122-134.
14. Jian M-Y, Liu Y, Li Q et al. N-cadherin coordinates AMP kinasemediated lung vascular repair. Amer J Physiol Lung Cell Mol Physiol 2016; 310 (1): L71-L85.

15. Chung S, Yao J, Suyama K et al. N-cadherin regulates mammary tumor cell migration through Akt3 suppression. Oncogene 2013; 32 (4): $422-430$.

16. Luan Y, Zhang L, Chao $S$ et al. Mesenchymal stem cells in combination with erythropoietin repair hyperoxia-induced alveoli dysplasia injury in neonatal mice via inhibition of TGF- $\beta 1$ signaling. Oncotarget 2016; 7 (30): 47082-47094.

17. Livak, Schmittgen. Analysis of relative gene expression data using real-time quantitative PCR and the $2^{-\Delta \Delta C t}$ method. Methods 2001; 25: 402-408.

18. Li L, Wu D. miR-32 inhibits proliferation, epithelial-mesenchymal transition, and metastasis by targeting TWIST1 in non-small-cell lung cancer cells. OncoTargets Ther 2016; 9: 1489-1498.

19. Zhao Z, Rahman MA, Chen ZG, Shin DM. Multiple biological functions of TWIST1 in various cancers. Oncotarget 2017; 8 (12): 2038020393.

20. Qin Q, Xu Y, He T, Qin C, Xu J. Normal and disease-related biological functions of TWIST1 and underlying molecular mechanisms. Cell Res 2012; 22 (1): 90-106.

21. Gajula RP, Chettiar ST, Williams RD et al. The Twist Box Domain is Required for TWIST1-induced Prostate Cancer Metastasis. Molecular cancer research: MCR 2013; 11 (11). doi: 10.1158/1541-7786.MCR-130218-T.

22. Ávila-Moreno F, Armas-López L, Álvarez-Moran AM et al. Overexpression ofMEOX2 and TWIST1 Is Associated with H3K27me3 Levels and Determines Lung Cancer Chemoresistance and Prognosis. Patnaik S, ed. PLoS ONE 2014; 9 (12): e114104.

23. Skrzypek K, Tertil M, Golda S et al. Interplay Between Heme Oxygenase-1 and miR-378 Affects Non-Small Cell Lung Carcinoma Growth, Vascularization, and Metastasis. Antioxidants Redox Signaling 2013; 19 (7): 644-660.

24. Ma J, Mannoor K, Gao L et al. Characterization of microRNA transcriptome in lung cancer by nex generation deep sequencing. Mol Oncol 2014; 8 (7): 1208-1219.

25. Geng Q, Fan T, Zhang B, Wang W, Xu Y, Hu H. Five microRNAs in plasma as novel biomarkers for screening of early-stage non-small cell lung cancer. Resp Res 2014; 15 (1): 149.

26. Wang W-X, Danaher RJ, Miller CS et al. Expression of miR-15/107 Family MicroRNAs in Human Tissues and Cultured Rat Brain Cells. Genom, ProteomBioinform 2014; 12 (1): 19-30.

Received September 21, 2017. Accepted October 19, 2017. 